\title{
Knowledge, attitude, and practice of family physicians regarding diabetic neuropathy in family practice centers: Suez Canal University
}

\section{Nadia Mabrouk, Mohamad Abdou', Hebattalha Nour-Eldin, Amira A. Solyman El-Foly, Seham Ahmad Omar', Hamdy Ahmad Sliem ${ }^{1}$}

Departments of Family Medicine and ${ }^{1}$ Internal Medicine, Faculty of Medicine, Suez Canal University, Ismailia, Egypt

Address for the Correspondence: Prof. Hamdy Ahmad Sliem, Suez Canal University Hospital, Ismailia-41522, Egypt. E-mail: Hamdy.sliem@yahoo.com

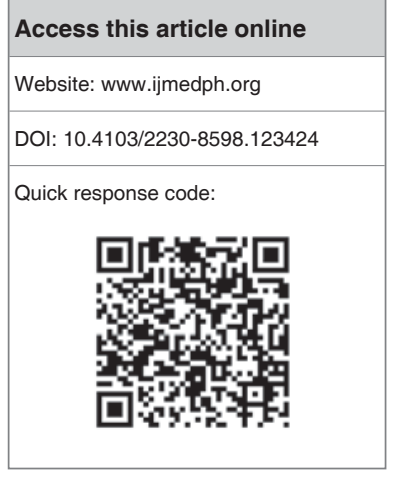

Background: Diabetic neuropathy (DN) can affect any part of the nervous system and should be suspected in all patients who have had diabetes for more than 5 years. Family physicians (FPs) can play an important role with the care and education of people with diabetes. They can augment the knowledge and motivate the diabetics to acquire a healthy life style, which would further lead to a good glycemic control providing protection from the chronic complications. Lack of compliance with the guidelines on the part of the diabetic subjects, indicates deficiencies in the FPs' knowledge, implementation techniques, and attitude problems. Therefore, the present study was conducted to assess FPs' knowledge, attitude, and practice regarding DN for further educational interventions that will improve their quality of care for diabetic patients in family practice centers. Materials and Methods: The study population was 60 FPs working in family practice centers affiliated to Suez Canal University Hospitals. The questionnaire composed of three groups of questions to collect data for evaluation of knowledge, attitude, and practice; two written patient problems to assess their practice and two questions to assess barriers and recommendations of physicians. To pass the evaluation; 50,80 , and $60 \%$ were the cut off points to pass the evaluation for knowledge, attitude, and practice, respectively. Results: 48.3 , 66.7 , and $43.3 \%$ of the evaluated FPs passed the knowledge, attitude, and practice assessment, respectively. Eighty-five percent of physicians felt that they need more knowledge and training in DN management. Physicians' qualification $(P=0.037)$ was a significant variable in passing the knowledge test, but qualification and experience years $(P=0.007$ and 0.035 , respectively) were significant variables in passing the practice test. There was a positive significant $(P=0.021)$ correlation between practice and knowledge score. Postgraduate knowledge accounts the majority $(78.3 \%)$ as a source of information about DN. Providing physicians with standardized guidelines $(81.7 \%)$, continuous medical education seminars $(76.7 \%)$ and training courses $(76.7 \%)$ came at the top of physician's recommendations to improve DN care by FPs. Conclusion: This study has identified the need for improvement in family medicine physician practices for treating and educating diabetics and recommended that awareness and educational programs are necessary to update the FPs on screening, effective treatment of diabetes and prevention of DN.

Key words: Attitude, diabetic neuropathy, family physicians, knowledge

\section{INTRODUCTION}

The World Health Organization (WHO) describes diabetes mellitus (DM) as the most common endocrine disease in the world. In the year 2000, diabetes affects more than 230 million people worldwide, and according to the most recent projections, it is expected to affect 370 million people by the year 2030 ${ }^{[1]}$ For Egypt, the total projected number of people with diabetes is about 6.7 million $(9 \%){ }^{[2]}$ Diabetic neuropathy (DN) is a debilitating disorder that occurs in nearly $50 \%$ of patients with diabetes. It is a late finding in type 1 diabetes, but can be an early finding in type 2 diabetes. Neuropathy is estimated to be present in $7.5 \%$ of patients at the time of diabetes diagnosis. ${ }^{[3-7]}$

DN can affect any part of the nervous system. This nerve disorder should be suspected in all the patients with type 2 diabetes and in patients who have had type 1 diabetes for more than 5 years. In 
some instances, patients with DN have few complaints, but their physical examination reveals mild to moderately severe sensory loss. Idiopathic neuropathy has been found to precede the onset of type 2 diabetes or to occur as an early finding in the disease. ${ }^{[8-11]}$ In the past 3 decades, despite considerable advances in treatment modalities of diabetes, it has been shown considerable gaps between patient's outcome and acceptable treatment in developed and also in developing countries.

Different reasons are proposed in failure to achieve therapeutic goals such as poor adherence to treatment regimens by patients or malpractice by physicians. ${ }^{[12]}$ It has been observed that in addition to physicians' knowledge; their attitude about treatment was important to achieve goals. In other words, physicians' belief was important factor in their success for treatment of diabetes. ${ }^{[13]}$ On the other hand, the knowledge and practice of diabetic patients to follow therapeutic principles showed a direct relationship with the attitude of physicians to diabetes care. This study was conducted to assess family physician's (FPs) knowledge, attitude, and practice regarding $\mathrm{DN}$ for further educational interventions that will improve their quality of care for diabetics.

\section{MATERIALS AND METHODS}

This study was designed as cross-sectional descriptive study including all FPs working in family medicine centers affiliated to Faculty of Medicine, Suez Canal University. The number of the physician was 60 . Only $18.3 \%$ of them were female, average age was 28.4 years, and $31.7 \%$ had medical experience for more than 5 years. These demographic data are presented in Table 1. There are nine family medicine centers distributed in Ismailia (Abo-khalifa, El-Mahsama, and Fanara), Port Said (Port-Fouad and El-Salam), and Suez (El-Omda, Amer, El-Gabaliat, and 24 October) Governorates.

All the FPs under training in (Diploma, Master degree, and Fellowship) working in family medicine practice centers (FMC) affiliated to Suez Canal University was included. The study population was subjected to self-administrative semi-structured questionnaire which was developed by the researchers, revised by two experts in the field of diabetes and community medicine and validated according to pilot study by $10 \mathrm{FPs}$. The questionnaire was composed of three parts:

A) Part I: Sociodemographic of FPs; including gender, age, qualification, experience years in family medicine, practice location, and the number of diabetic patients seen per week; in addition to some questions regarding their opinions on sources of their knowledge about diabetes and their perception regarding their knowledge and training in $\mathrm{DN}$.

B) Part II: Multiple choice questions and two patient problems for assessing knowledge, attitude, and practice of FPs regarding DN. The scoring system was:

\begin{tabular}{|c|c|}
\hline Items & Results \\
\hline \multicolumn{2}{|l|}{ Age (years): } \\
\hline$<30$ & $65 \%(n=39)$ \\
\hline$>30$ & $35 \%(n=21)$ \\
\hline \multicolumn{2}{|l|}{ Gender: } \\
\hline Male & $81.7 \%(n=49)$ \\
\hline Female & $18.3 \%(n=11)$ \\
\hline \multicolumn{2}{|l|}{ Medical experience: } \\
\hline$<5$ years duration & $68.3 \%(n=41)$ \\
\hline$>5$ years duration & $31.7 \%(n=19)$ \\
\hline \multicolumn{2}{|l|}{ Qualification: } \\
\hline Qualified (MSc degree) & $35 \%(n=39)$ \\
\hline Involved in training programs & $65 \%(n=21)$ \\
\hline
\end{tabular}

$n=$ Number of studied physician

- Knowledge score: One point for each correct answer of total 15 points. The cut off point to pass the evaluation for knowledge considered $50 \%$ for correct answers of the total score. Not passed knowledge if $<50$ and passed knowledge if $\geq 50$.

- Attitude score: A score of 5 was given for "strongly agree", 4 for "agree", 3 for "unsure", 2 for "disagree", and 1 for "strongly disagree"; of total 20 points. The cut off point to pass the evaluation for attitude considered $80 \%$ for correct answers of the total score. Favorable attitude if $\geq 80 \%$ and unfavorable attitude if $<80 \%$.

- Practice score: A score of 3 was given for "always", 2 for "sometimes", 1 for "rarely", and 0 for "never"; giving total of 60 points. Also, one point for each correct answer of total 6 points in the patient problems. The total practice scoring was 66 points. The cut off point to pass the evaluation for practice considered $60 \%$ for correct answers of the total score. Appropriate if $\geq 60 \%$ and inappropriate if $<60 \%$.

C) Part III: Two questions about the greatest barriers faced by physicians in DN management and their recommendations to improve their practice at family medicine level.

\section{Pilot study}

The questionnaire was pretested on FPs before the beginning of data collection to determine if the questions asked were understood by the respondents or not, perform any modifications needed, and determine the required time for the questionnaire.

\section{Data analysis}

The data were coded and organized. The final study results were stated by using the Statistical Package for Social Sciences (SPSS) program, version 15. Statistical significance was considered at $P$-values $<0.05$ and a higher significance at $P$-values $<0.001$. 


\section{Ethical consideration}

The study protocol was approved by the faculty committee of research ethics. The data were collected after taking a written consent from the study participants and their names were omitted (anonymous). All data were confidential and used only for scientific research purposes.

\section{RESULTS}

Results of physicians' knowledge, attitude, and practices are presented in Table 2. 48.3, 66.7, and 43.3\% of the evaluated FPs passed the knowledge, attitude, and practice assessment, respectively. Also, $85 \%$ of physicians felt that they need more knowledge and training in DN management. FPs qualification was a significant variable in passing the knowledge test $(P=0.037)$, but qualification and experience years $(P=0.007$ and 0.035 , respectively) were significant variables in passing the practice test, while no statistically significant relation was observed between sociodemographic characteristics and attitude scoring. There was a positive, significant $(P=0.021)$ correlation between practice score and knowledge score.

The physicians' recommendations are summarized in Table 3. Providing physicians with standardized guidelines, continuous medical education seminars, and training courses came at the top of recommendations to improve DN care by FPs.

\section{DISCUSSION}

Out of $60 \mathrm{FPs}, 65 \%$ of them aged less than 30 years old and $68.3 \%$ had less than 5 years' experience in clinical practice. This is not consistent with a study of Shera et al., conducted in Pakistan, 2002; about diabetes related knowledge, attitude, and practices of FPs. They demonstrated the average age of physicians as 42.18 years and the mean duration of clinical practice as 13.41 years. ${ }^{[14]}$

\begin{tabular}{lc} 
Table 2: Knowledge, attitude, and practice results \\
\hline Items & Results \\
\hline Knowledge: & \\
$\quad$ Passed & $48.3 \%(n=29)$ \\
$\quad$ Not passed & $51.7 \%(n=31)$ \\
Attitude: & \\
$\quad$ Favorable & $66.7 \%(n=40)$ \\
$\quad$ Nonfavorable & $33.3 \%(n=20)$ \\
Practice: & \\
$\quad$ Appropriate & $43.3 \%(n=26)$ \\
$\quad$ Inappropriate & $56.7 \%(n=34)$ \\
\hline$n=$ Number of studied physician &
\end{tabular}

\begin{tabular}{lc} 
Table 3: Physicians' recommendations to improve \\
diabetic care & Results \\
\hline Items & $81.7 \%(n=49)$ \\
\hline Providing standardized guidelines & $76.7 \%(n=46)$ \\
Continuous medical education seminars & $76.7 \%(n=46)$ \\
Training courses & \\
\hline$n=$ Number of studied physician
\end{tabular}

Also, the present study demonstrates that female physicians are the majority (81.7\%); while males form only 18.3\%. This is inconsistent with a study of Khan et al., conducted in $\mathrm{Al}$ Hasa district of Saudi Arabia, 2010; about knowledge, attitude, and practice of primary health care physicians in the management of type $2 \mathrm{DM}$, which demonstrate that the number of male physicians was much higher than that of female physicians: 72.73 and $27.27 \%$, respectively. ${ }^{[15]}$

In addition, there was about one-third qualified FPs; while 65\% physicians are involved in the training programs of (diploma, master, and fellowship), with no other specialties included in the study except FPs. This is inconsistent with a study of Peimani et al., conducted in Iran, 2010; about knowledge, attitude, and practice of physicians in the field of diabetes and its complications. They showed that about $37.7 \%$ of the participants were general physicians, while 15.9 and $46.4 \%$ were internist and other specialties, respectively. ${ }^{[16]}$

The current study demonstrates that about 48.3 and $43.4 \%$ of the studied FPs have good knowledge and practice scores, respectively regarding DN management. However, these results showed better knowledge and practice scores than a similar same study of Peimani et al., which revealed that $29 \%$ of total physicians had good knowledge score in the field of diabetes and its complications and $36.2 \%$ of physicians had acceptable practice in control and prevention of diabetes complications. ${ }^{[16]}$

The difference between these results and the current results may be explained by the availability of continuous educational programs for postgraduate FPs that has been conducted regularly in the Family Medicine Department of Suez Canal University.

By studying some factors affecting the results (passed or not passed) of FPs, the current data revealed that qualification of FPs is the main factor significantly affecting their knowledge results, while qualification and experience years in family medicine significantly affecting their practice results $(P<0.05)$.

As well, FPs with experience $\geq 5$ years significantly scored better in the practice segment than those with experience less than 5 years $(P=0.035)$, but there is no statistically significant relations in the segments of knowledge and attitude $(P=0.313$ and 0.844 , respectively). This is inconsistent with the study of Shera $e t$ al. ${ }^{[14]}$ They demonstrated that, doctors with practice duration of 6-10 years provided more correct answers in the segment of knowledge and attitude, than those with either less or more $(P=0.01)$. Also inconsistent with the study of Khan et al., demonstrated that the means of overall scores were significantly higher for physicians with 1-5 years of experience, than those with $>5$ years of practice $(P<0.05) \cdot{ }^{[15]}$

The present study demonstrates that there is no difference between FPs practicing in the urban areas and those practicing in the rural areas in the attitude segment (50 vs $50 \%, P=0.715$ ), but there is a slight difference in the knowledge part ( 51.7 vs $48.3 \%, P=0.993$ ), and in the practice part (38.5 vs $61.5 \%, P=0.073)$. These however 
did not achieve any statistical significance. This is inconsistent with the study of Shera et al. ${ }^{[14]}$ that demonstrated doctors practicing in the urban areas gave more correct answers in the segment of attitude (53 vs $43 \%, P=0.05$ ) and practice (52 vs $41 \% P=0.03$ ). In the knowledge variable, the doctors of the rural areas scored better (62 vs $56 \% P=0.2)$. Also inconsistent with the study of Khan et al., ${ }^{[15]}$ which demonstrated that the scores of rural general practitioners (GPs) were higher than that of GPs practicing in urban areas, which was statistically significant $(P=0.003)$.

The difference between these results and the current results may be explained that there is no fixed place for work and our physicians are in rotation all the time with some minimal exceptions. Also our physicians working in the rural and urban areas undergo the same educational programs and the same courses and modules that applied to postgraduate FPs who attend weekly in the scientific day in the Family Medicine Department of Suez Canal University.

In the present study, postgraduate knowledge form the commonest source for DN knowledge (78.3\%), followed by internet $(51.7 \%)$, and text books (50\%); while dated papers from journals, courses or activities, and conferences and meetings accounts only for 31.7, 26.7 , and $25 \%$, respectively. This is inconsistent with the study of Khan et al., which demonstrated that the source of information of the GPs on diabetes management was the clinical practice guidelines (15.2\%), scientific meetings (12.1\%), educational programs (19.2\%), and medical journals $(2 \%){ }^{[15]}$ Also inconsistent with the study of Peimani et al., that demonstrated they did not have any information about whether the physicians had experiences in diabetes clinics, or they participated in workshops and diabetes training courses as a source of information. ${ }^{[16]}$

Concerning the physicians' self-reported barriers regarding DN management, the present study shows that the highest percentage of FPs agree with barriers focused on inadequate physician tools regarding diabetic peripheral neuropathy assessment (80\%), while $88.3 \%$ disagree that diabetic patients refuse examinations to assess neuropathy. Other studies show that poor practice of physicians is associated with extensive health problems in the community such as increasing the incidence of medical errors, patient dissatisfaction, lack of control of many chronic diseases, delay in diagnosis, and the illegitimate use of drugs. ${ }^{[14,17,18]}$

Concerning to the physicians' recommendations to improve DN management in family practice centers, the majority (81.7\%) of their recommendations focused on providing physicians with standardized guidelines, while $76.7 \%$ focused on continuous medical education seminars and training courses. The role of the healthcare provider, in the case of chronic illness is different than that of seasonal, episodic, and temporary ailments. For the successful treatment of a diabetic, the FPs have to acquire the understanding, cooperation, and involvement of other family members. ${ }^{[19]}$ This will demand more of the doctor's time along with a sympathetic approach. The ideal method would be a team work, where the education of the diabetics is shared and coordinated by medical education and continuing medical education programs play an important role in enabling the healthcare providers to treat diabetics in a most efficient and economical manner. ${ }^{[20]}$

Sample size is the main limitation of the study, while the variations of participants and work places are the main strength. However, further studies are required with a relatively larger sample size to detect the impact of the current data on the quality of diabetes health care delivered by FPs for DN.

\section{CONCLUSION AND RECOMMENDATION}

About half of the FPs passed the knowledge and have favorable attitude, while about two-thirds have appropriate practice regarding DN. Providing FPs with standardized guidelines, continuous medical education seminars and training courses came at the top of physician's recommendations to improve DN care by FPs. This study has identified the need for further improvement in family medicine physician practices for treating and educating diabetics and recommended that awareness and educational programs are necessary to update the FPs on screening, effective treatment of diabetes, and prevention of DN. This should be the scope of any future health plans and programs for target populations.

\section{REFERENCES}

1. Wild S, Roglic G, Green A, Sicree R, King H. Global prevalence of diabetes: Estimates for the year 2000 and projections for 2030. Diabetes Care 2004;27:1047-53.

2. Arafa NA, Amin GE. The epidemiology of diabetes mellitus in Egypt: Results of a National Survey. Egypt J Community Med 2010;28:29-37.

3. Dyck PJ, Kratz KM, Karnes JL, Litchy WJ, Klein R, Pach JM, et al. The prevalence by staged severity of various types of DN, retinopathy, and nephropathy in a population-based cohort: The Rochester Diabetic Neuropathy Study. Neurology 1993;43:817-24.

4. Partanen J, Niskanen L, Lehtinen J, Mervaala E, Siitonen O, Uusitupa M. Natural history of peripheral neuropathy in patients with non-insulindependent diabetes mellitus. N Engl J Med 1995;333:89-94.

5. Novella SP, Inzucchi SE, Goldstein JM. The frequency of undiagnosed diabetes and impaired glucose tolerance in patients with idiopathic sensory neuropathy. Muscle Nerve 2001;24:1229-31.

6. Singleton JR, Smith AG, Bromberg MB. Increased prevalence of impaired glucose tolerance in patients with painful sensory neuropathy. Diabetes Care 2001;24:1448-53.

7. Perkins BA, Olaleye D, Bril V. Carpal tunnel syndrome in patients with diabetic polyneuropathy. Diabetes Care 2002;25:565-9.

8. Mondal A, Sen S, Chanda D, Kundu S, Chatterjee M, Mukherjee S. Evaluation of diabetic polyneuropathy in type 2 diabetes mellitus by nerve conduction study and association of severity of neuropathy with serum sFasL level. Indian J Endocrinol Metab 2012;16(Suppl 2):S465-7.

9. Alrawahi AH, Rizvi SG, Al-Riyami D, Al-Anqoodi Z. Prevalence and risk factors of diabetic nephropathy in omani type 2 diabetics in Al-dakhiliyah region. Oman Med J 2012;27:212-6.

10. Kote GS, Bhat AN, K T, Ismail MH, Gupta A. Peripheral insensate neuropathy-is height a risk factor? J Clin Diagn Res 2013;7:296-301.

11. Hirose T. Management of diabetic neuropathy. Masui 2010;59:1378-84.

12. Murugesan N, Shobana R, Snehalatha C, Kapur A, Ramachandran A. Immediate impact of diabetes training programme for primary care physicians--an endeavor for national capacity building for diabetes management in India. Diabetes Res Clin Pract 2009;83:140-4.

13. Weinberger M, Cohen SJ, Mazzuca SA. The role of physicians' knowledge and attitudes in effective diabetes management. Soc Sci Med 1984;19:965-9. 
14. Shera AS, Jawad F, Basit A. Diabetes related knowledge, attitude and practices of family physicians in Pakistan. J Pak Med Assoc 2002;52:46570.

15. Khan AR, Al Abdul Lateef ZN, Khamseen MB, Al Aithan MA, Khan SA, Al Ibrahim I. Knowledge, attitude and practice of ministry of health primary health care physicians in the management of type 2 diabetes mellitus: $A$ cross-sectional study in the Al Hasa District of Saudi Arabia, 2010. Niger J Clin Pract 2011;14:52-9.

16. Peimani M, Tabatabaei MO, Heshmat R, Amiri MS, Sanjari M, Pajouhi M. Knowledge, attitude and practice of physicians in the field of diabetes and its complications; a pilot study. Iran J Diabetes Lipid Disord 2010;9:1-7.

17. Wong T, Foote EF, Lefavour GS, Cody RP, Brown CJ, Sherman RA. Physician knowledge and practice patterns relating to diabetic nephropathy. J Am Pharm Assoc (Wash) 1999;39:785-90.

18. Dehghan G, Mahmudian A, Jamshidi A, Hormozi M, Asgari H. The effect of education of patient management model on physicians practice. TabibE-Shargh J 2007;9:217-24.

19. Hehseth LD, Susman JL, Crabtree BF, O'Connor PJ. Primary care physicians' perception of diabetes management. A balancing act. J Fam Pract 1999;48:37-42.

20. Stein LS. The effectiveness of continuing medical education: Eight research reports. J Med Educ 1981;56:103-10.

How to cite this article: Mabrouk N, Abdou M, Nour-Eldin $\mathrm{H}$, Solyman El-Foly AA, Omar SA, Sliem HA. Knowledge, attitude, and practice of family physicians regarding diabetic neuropathy in family practice centers: Suez Canal University. Int J Med Public Health 2013;3:230-4.

Source of Support: Nil, Conflict of Interest: None declared. 